\title{
Magnet-assisted Flow Cytometry of in vivo Tumors to Quantitate Cell-specific Responses to Magnetic Iron Oxide Nanoparticles \\ Preethi Korangath ${ }^{1, *}$ and Robert Ivkov ${ }^{1,2,3,4}$
}

\begin{abstract}
${ }^{1}$ Dept of Radiation Oncology and Molecular Radiation Sciences, School of Medicine, Johns Hopkins University, Baltimore, MD 21231 USA; ${ }^{2}$ Department of Oncology, School of Medicine, Johns Hopkins University, Baltimore, MD 21231 USA; ${ }^{3}$ Department of Mechanical Engineering, Whiting School of Engineering, Johns Hopkins University, Baltimore 21218, USA; ${ }^{4}$ Department of Materials Science and Engineering, Whiting School of Engineering, Johns Hopkins University, Baltimore 21218, USA *For correspondence: kpreeth1@jh.edu
\end{abstract}

[Abstract] A clear understanding of nanoparticle interactions with living systems at the cellular level is necessary for developing nanoparticle-based therapeutics. Magnetic iron oxide nanoparticles provide unique opportunities to study these interactions because of their responsiveness to magnetic fields. This enables sorting of cells containing nanoparticles from in vivo models. Once sorted, flow cytometry can identify individual cell types, which can be further analyzed for iron content, gene or protein expression changes associated with nanoparticle uptake, and for other biological responses at a molecular level. Here we provide a detailed protocol to sort and identify cells in the tumor microenvironment that have internalized magnetic iron oxide nanoparticles following intravenous administration.

Keywords: Flow cytometry, Iron oxide nanoparticles, Magnetic separation, Systemic injection

[Background] Several nanoparticle-based cancer medicines are in clinical use because they have demonstrated safety and efficacy for diagnosis, cancer drug delivery, and hyperthermia (Marchal et al., 2015; Wu et al., 2015). Yet, there remain critical gaps in our understanding of cancer nanomedicine, largely because details of interactions between nanoparticles and immune cells remain unknown. Others and we have identified nanoparticle-immune cell interactions as critical variables affecting in vivo nanoparticle fate and retention in tumors (Sheen et al., 2014; Zanganeh et al., 2016; Korangath et al., 2020). Flow cytometry enables quantitative analytical sorting of mixed cell populations by combining light scattering with detection of a fluorescent signal from appropriately labeled cells. To track nanoparticle uptake in cells, the nanoparticles are often labeled with a fluorescent dye to enable detection. The presence of dyes or other 'excipient' molecules on nanoparticles can change nanoparticle pharmacokinetics and biodistribution in unintended and unrealized ways, and do not provide sufficient analyte for quantitative validation of nanoparticle concentration. Here we demonstrate the use of the magnetic property of unlabeled iron oxide nanoparticles (no fluorescence labeling) taken up by the cells, when administered intravenously. Our developed assay provides quantitative and cell-specific data of unaltered nanoparticle uptake following systemic (i.e., intravenous) delivery. Our assay exploits the directional response of magnetic iron oxide nanoparticles (MIONs) to high-gradient magnetic fields, which causes cells containing the MIONs to sediment near the magnet, whereas cells devoid of MIONs 
remain in supernatant. In this manner, cells are 'pre-sorted' by magnetic separation to differentiate between the two populations before flow cytometry analysis. Following sorting, cells can be analyzed further by inductively coupled plasma mass spectrometry (ICP MS) or ferene-s assay (Hedayati et al., 2018) to validate concentration of the analyte $(\mathrm{Fe})$ which is then related to intracellular nanoparticle content; and, by other gene- or protein-based array technologies to assess biological responses at the molecular level within cells (Figure 1).

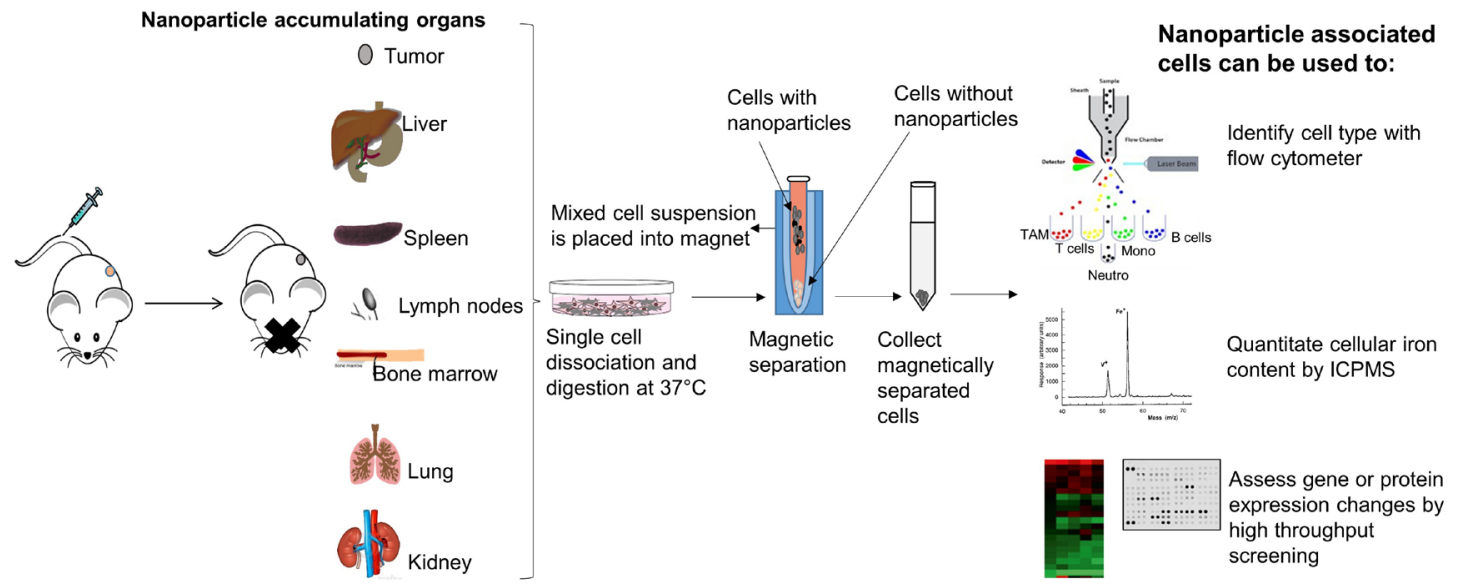

Figure 1. Concept of magnet-assisted cell isolation for detailed downstream analysis. When injected into the tail vein, iron oxide nanoparticles accumulate in organs and tumor. After harvest, tissues can be dissociated into single cells and nanoparticle-associated cells can then be isolated by placing the entire mixture on a permanent rare earth magnet. Cells associated with the iron oxide nanoparticles (internal or membrane bound) will separate from the suspension and attach to the tube wall proximal to the magnet. Cells not associated with a significant concentration of nanoparticles will remain in suspension (supernatant). Magnetically sorted cells can then be further analyzed with a flow cytometer to distinguish among cell types or lineages. Absolute amount of iron in cells can be determined by inductively coupled plasma mass spectrometry (ICPMS), or by other quantitative iron assays. Nanoparticle-induced changes in cells at the molecular level can be identified by further analysis using gene- or protein-based array technologies. Comparisons with cells not associated with the nanoparticles is also possible by collecting and analyzing the supernatant.

\section{Materials and Reagents}

1. Micropipette tips (Rainin, catalog numbers: 17005860 [20 $\mu \mathrm{l}] ; 30389240$ [200 $\mu \mathrm{l}] ; 30389213$ $[1,000 \mu l])$

2. Corning ${ }^{\circledR}$ non-treated culture dishes $(\mathrm{D} \times \mathrm{H} 100 \mathrm{~mm} \times 20 \mathrm{~mm})$ (Corning, catalog number: 430591)

3. Syringe (3 ml) (Fisher Scientific, catalog number: 14-823-436)

4. Conical tube $(50 \mathrm{ml})$ (Thermo Scientific, catalog number: 339653) 
5. Cell strainer Nylon (100 $\mu \mathrm{m})$ (BD Falcon, catalog number: 352360)

6. Aluminum foil

7. Integra ${ }^{\mathrm{TM}}$ Miltex $^{\mathrm{TM}}$ Sterile Surgical Blades (Fisher Scientific, catalog number: 12-460-444)

8. Microcentrifuge tubes (Fisher Scientific, catalog number: 05-408-137)

9. Falcon ${ }^{\mathrm{TM}}$ Round-Bottom Polystyrene Tubes (Flow tubes) (Corning, catalog number: 352054 )

10. ACK lysing buffer (Quality Biologicals, catalog number: 118-156-101)

11. Dulbecco's Modified Essential Medium (DMEM) high glucose (MilliporeSigma, catalog number: D5796)

12. Heat Inactivated FBS (HI-FBS) (Fisher Scientific, catalog number: $\mathrm{SH} 30109.03 \mathrm{HI})$

13. Collagenase Type 4 (Worthington Biochemical Corporation, catalog number: CLS4)

14. Hyaluronidase (MP Biomedicals, catalog number: 02151272)

15. Phosphate buffered saline (PBS) (Corning Cellgro, catalog number: 21-040-CV)

16. OneComp eBeads (eBioscience, catalog number: 01-1111-42)

17. eBioscience ${ }^{\mathrm{TM}}$ Fixation/Permeabilization Concentrate (Thermo Scientific, catalog number: 005123-43)

18. eBioscience ${ }^{\mathrm{TM}}$ Fixation/Permeabilization Diluent (Thermo Scientific, catalog number: 00-522356)

19. Anti CD16/32 antibody (Fc blocker) (BioLegend, catalog number: 101302), dilution 1:100

20. Zombie Aqua (Live/dead stain) (BioLegend, catalog number: 423102), dilution 1:100

21. Anti CD45- APC-Cy7 antibody (BioLegend, catalog number: 103116), dilution 1:500

22. Anti MHCII- AF700 antibody (BioLegend, catalog number: 107622), dilution 1:200

23. Anti CD3- BV711 antibody (BioLegend, catalog number: 100241), dilution 1:500

24. Anti TCRyס- PE antibody (BioLegend, catalog number: 118108), dilution 1:1,000

25. Anti CD19- BV650 antibody (BioLegend, catalog number: 115541), dilution 1:500

26. Anti CD49b- PERCP Cy5.5 (BioLegend, catalog number: 108916), dilution 1:200

27. Anti CD11b- BV421 antibody (BioLegend, catalog number: 101235), dilution 1:200

28. Anti Ly6C- APC antibody (BioLegend, catalog number: 128016), dilution 1:200

29. Anti Ly6G- FITC antibody (BioLegend, catalog number: 127606), dilution 1:500

30. Anti F4/80- BV605 antibody (BioLegend, catalog number: 123133), dilution 1:200

31. Anti CD11c- PECy7 antibody (BD Pharmingen, catalog number: 561022), dilution 1:500

32. Anti Cytokeratin-PE antibody (Abcam, catalog number: Ab52460), dilution 1:500

33. Ice

34. Dimethyl sulfoxide (DMSO)

\section{Equipment}

1. Micropipettes

2. Tube holders and racks

3. Ice holder 
4. Biosafety cabinet (BSL2)

5. Rotator (Fisher Scientific, UVP HB-1000 Hybrodizer Hybridization Oven, catalog number: UVP 95003001)

6. Centrifuge (Eppendorf, model: $5810 \mathrm{R})$

7. Rare earth permanent magnet (Promega, Magnesphere ${ }^{\circledR}$ Technology magnetic separation stand, catalog number: Z5343)

8. Incubator $37^{\circ} \mathrm{C}$ with $5 \% \mathrm{CO}_{2}$ (Thermo Scientific, Heracell 160l, model: 41774412)

9. Nexcelom cell counter (Nexcelom Bioscience, Cellometer auto T4 automated cell counter, model: Auto T4-003-0489)

10. Flow cytometer (BD Biosciences, model: LSR-II)

\section{Software}

1. BD FACSDiva software (BD Biosciences, CA, USA)

2. Flowjo software version 10 (BD Biosciences, $\mathrm{CA}, \mathrm{USA}$ )

\section{Procedure}

A. Preparation of digestion media

1. Prepare digestion media just before use. Fresh media has to be used for each experiment. To DMEM high glucose media, add 10\% heat inactivated FBS (HI-FBS), $0.1 \%$ collagenase Type 4 and $0.005 \%$ hyaluronidase. Both collagenase Type 4 and hyaluronidase are in powder form, weigh and add directly to the media. Mix with gentle agitation until all ingredients dissolve. Aliquot $25 \mathrm{ml}$ to each conical tube for each sample.

B. Sample preparation

1. Euthanize animals previously injected with iron oxide nanoparticles. Excise tumors and record their weight.

2. Mince completely with a razor blade in a Petri dish.

3. Use a syringe plunger to mash the tumor.

4. Transfer to the conical tube containing $25 \mathrm{ml}$ digestion media $(25 \mathrm{ml}$ per $150-200 \mathrm{mg}$ tumor weight).

5. Rotate at $37^{\circ} \mathrm{C}$ for $30 \mathrm{~min}$ and filter through a $100 \mu \mathrm{m}$ filter while mashing with a syringe plunger.

6. Centrifuge at $335 \times g$ at $23^{\circ} \mathrm{C}$ for $10 \mathrm{~min}$.

7. Wash with $25 \mathrm{ml}$ DMEM/tumor with $10 \% \mathrm{HI}-\mathrm{FBS}$. Resuspend with a pipette and centrifuge again at $335 \times \mathrm{g}$ at $23^{\circ} \mathrm{C}$ for $10 \mathrm{~min}$.

8. Discard the supernatant and add $3 \mathrm{mIACK}$ (Ammonium-Chloride-Potassium) lysing buffer. ACK lysing buffer is used to lyse red blood cells from flow preparation. It is commercially available, 
but can also be prepared with ammonium chloride, potassium bicarbonate, and sodium EDTA. Resuspend with a pipette and leave at room temperature for 3-5 $\mathrm{min}$.

9. Add DMEM with $10 \% \mathrm{HI}-\mathrm{FBS}$ up to $25 \mathrm{ml}$ and centrifuge at $335 \times \mathrm{g}$ at $23{ }^{\circ} \mathrm{C}$ for $10 \mathrm{~min}$ and discard the supernatant.

C. Magnetic separation

1. Add $4 \mathrm{ml}$ DMEM with $10 \%$ HI-FBS.

2. Resuspend and transfer all $4 \mathrm{ml}$ to labeled flow tubes.

3. Keep all tubes upright on a rare earth permanent magnet and incubate for at least $30 \mathrm{~min}$ in $37{ }^{\circ} \mathrm{C}$ incubator with $5 \% \mathrm{CO}_{2}$.

4. After magnetic separation, carefully collect supernatant into another tube.

5. Add $4 \mathrm{ml}$ of DMEM with $10 \%$ HI-FBS to the magnetically attached fraction and resuspend.

6. Centrifuge all tubes at $335 \times g$ at $23^{\circ} \mathrm{C}$ for $10 \mathrm{~min}$ and discard supernatant. A schema of the method is given in Figure 2 .

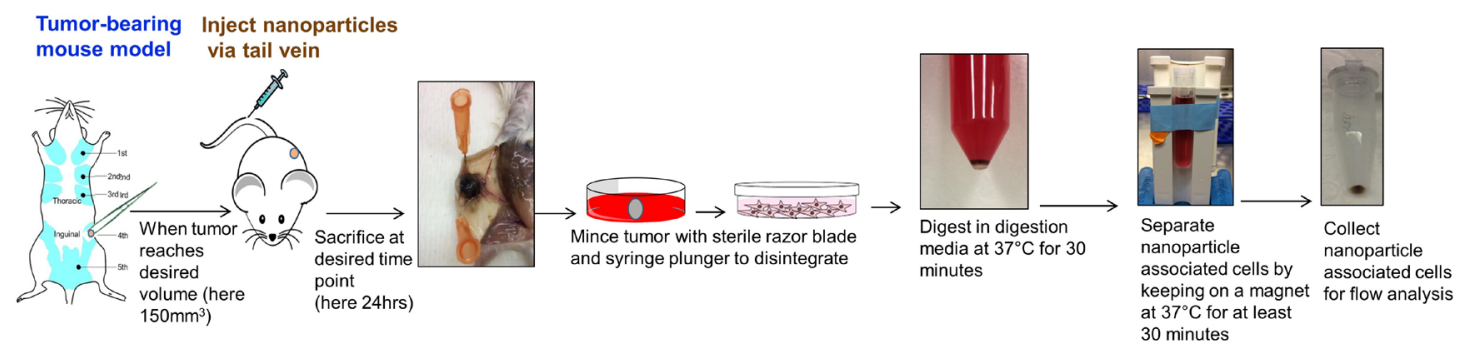

Figure 2. Overall scheme of magnet-assisted cell sorting from tumor for flow cytometry

D. Staining and fixation

1. Wash sedimented cells (pellet) with $2 \mathrm{ml}$ of PBS by resuspending using a pipette.

2. Centrifuge at $335 \times g$ at $23{ }^{\circ} \mathrm{C}$ for $10 \mathrm{~min}$.

3. Add adequate amount of PBS (usually $1 \mathrm{ml}$ ) to enable cell counting, and count cells after dilution.

4. Transfer 2 million cells from each tube and into fresh tubes.

5. Separately, reserve 1-2 million cells for unstained control, live/dead control and for each FMO tubes (FMO: Fluorescence Minus One) for compensation and gating.

6. Centrifuge at $335 \times g$ at $23^{\circ} \mathrm{C}$ for $5 \mathrm{~min}$.

7. Discard the supernatant and add $100 \mu \mathrm{l}$ live/dead solution (stock in DMSO) $(1 \mu \mathrm{l} / 100 \mu \mathrm{l}$ PBS) and incubate at room temperature in dark for $30 \mathrm{~min}$.

8. Centrifuge at $335 \times g$ at $23^{\circ} \mathrm{C}$ for $3 \mathrm{~min}$.

9. Wash with $100 \mu$ l PBS by resuspending with a pipette and centrifuge at $335 \times g$ at $23{ }^{\circ} \mathrm{C}$ for 3 $\min$.

10. Discard the supernatant and add $100 \mu \mathrm{Fc}$ blocker $(1 \mu \mathrm{l} / 100 \mu \mathrm{l}$ PBS) and incubate on ice for 10 $\min$.

11. Centrifuge at $335 \times g$ at $23^{\circ} \mathrm{C}$ for $3 \mathrm{~min}$. 
12. Wash with $100 \mu \mathrm{I}$ PBS with $5 \% \mathrm{HI}-\mathrm{FBS}$ by resuspending with a pipette.

13. Centrifuge at $335 \times g$ at $23^{\circ} \mathrm{C}$ for $3 \mathrm{~min}$.

14. Add one drop of single stain beads to new tubes without cells.

15. Prepare antibody cocktails $(50 \mu \mathrm{l} / \mathrm{sample})$ in biosafety cabinet in dark

16. All dilutions are made in PBS with $5 \%$ HI-FBS on ice:

a. For test samples-add all antibodies in appropriate dilution.

b. For single stain beads-single antibody in appropriate dilution.

c. For FMO-all antibodies minus one antibody in appropriate dilution (e.g., if using 12 antibodies, 12 separate antibody cocktails should be prepared each with all 11 antibodies without 1 antibody).

17. Add $50 \mu \mathrm{l}$ of the antibody cocktail to their respective tubes, and mix gently with pipette and incubate at room temperature in dark for $30 \mathrm{~min}$.

18. Centrifuge at $335 \times g$ at $23^{\circ} \mathrm{C}$ for $3 \mathrm{~min}$.

19. Wash with $100 \mu \mathrm{l} \mathrm{PBS}$ with $5 \% \mathrm{HI}-\mathrm{FBS}$ by resuspending using a pipette.

20. Centrifuge at $335 \times g$ at $23^{\circ} \mathrm{C}$ for $3 \mathrm{~min}$.

21. Prepare fixation/ permeabilization (fix/perm) solution by mixing 1 part of concentrate with 3 parts of diluent as per manufacture protocol.

22. Resuspend in $50 \mu \mathrm{l}$ of fix/perm solution and incubate at room temperature in dark for $30 \mathrm{~min}$.

23. Centrifuge at $335 \times g$ at $23^{\circ} \mathrm{C}$ for $3 \mathrm{~min}$.

24. Wash with $100 \mu \mathrm{l}$ PBS with $5 \% \mathrm{HI}-\mathrm{FBS}$ by resuspending using a pipette.

25. Centrifuge at $335 \times g$ at $23^{\circ} \mathrm{C}$ for $3 \mathrm{~min}$.

26. Discard the supernatant and add $200 \mu \mathrm{l}$ PBS with $5 \%$ HI-FBS. Run samples in flow cytometer LSR-II (BD Biosciences, San Jose, CA).

27. Can be stored in dark at $4{ }^{\circ} \mathrm{C}$ covered with aluminum foil for running next day (within $24 \mathrm{~h}$ ).

E. Data Acquisition

1. Acquire data in LSR II using BD FACSDiva software. First, run single stain controls to set up negative and positive gates. Then run FMOs and samples to record data collected from minimum of 200,000 events per sample. Export data as FCS files after compensation in BD FACSDiva software (Staats et al., 2019).

\section{Data analysis}

1. Use Flowjo software for analyzing data.

2. Export FCS files to Flowjo software and group them as FMOs and Tumor samples.

3. Open one FMO file and plot SSC-A vs. FSC-A.

4. Gate on all cells-P1.

5. Select P1 and plot FSC-H vs FSC-A to select single cells-P2.

6. Select P2 and plot SSC-A vs Live/dead to exclude dead cells. Gate on negative population-P3 
7. Select P3 and plot Live/dead vs. CD45+ve population. Use CD45 FMO to set up gate. Here in CD45 FMO, the signal for CD45 will be negative. This helps to set up gates in unbiased manner. Likewise, set up gates based on FMOs for all other colors. Use the same set up for all the samples by dragging the all samples gate of final set up to tumor samples group.

8. Identify cell populations based on the specific markers as given below. Gating strategy is given in Figure 3 and in Figure S8 of original paper (Korangath et al., 2020).

a. Tumor cells-CD45-ve, CK+ve

b. T cells-CD45+ve, CD3+ve

c. Gamma delta T cells-CD45+ve, CD3+ve, TCR+ve

d. B cells-CD45+ve, CD3-ve, CD19+ve

e. NK cells-CD45+ve, CD3-ve, CD49b+ve

f. Neutrophils-CD45+ve, CD3-ve, CD49b-ve, CD19-ve, CD11b+ve, Ly6G+ve

g. Dendritic cells-CD45+ve, CD3-ve, CD49b-ve, CD19-ve, MHC II+ve, CD11c+ve, F4/80-ve

h. Monocytes-CD45+ve, CD3-ve, CD49b-ve, CD19-ve, CD11b+ve, Ly6G-ve, Ly6C+ve, F4/80-ve

i. Tumor Associated Macrophages-CD45+ve, CD3-ve, CD49b-ve, CD19-ve, CD11b+ve, Ly6G-ve, Ly6C-ve, F4/80+ve

*Two separate tubes of same samples were used for tumor cell (Live/dead, CD45, CK antibodies) and immune subpopulation (Live/dead, CD45, CD3, CD49b, CD19, CD11b, Ly6G, Ly6C, F4/80, MHC II, CD11c, TCR antibodies) identification.
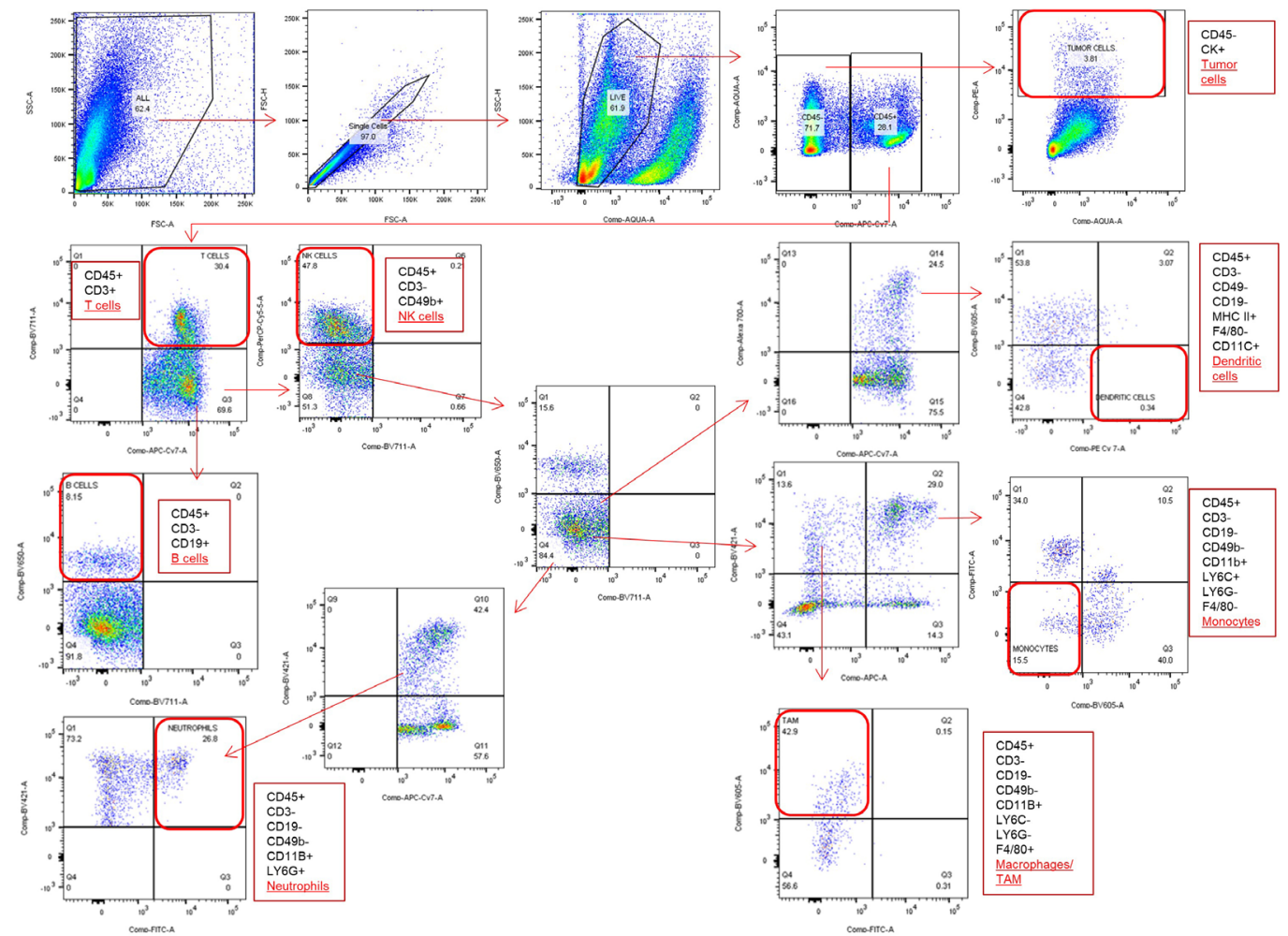

Figure 3. Flow cytometry gating strategy to identify different cell population 


\section{Acknowledgments}

This protocol is derived from a published study (Korangath et al., 2020) funded by the Jayne Koskinas Ted Giovanis Foundation for Health and Policy. In addition, partial support from $\mathrm{NIH}$ 5R25GM109441 and $\mathrm{NIH} / \mathrm{NCl}$ grant P30 CA006973 were used. Authors wish to acknowledge Ms. Tricia $L$ Nilles for assistance in flow cytometry experiments.

\section{Competing interests}

R.I. is an inventor on nanoparticle patents. All patents are assigned to either The Johns Hopkins University or Aduro BioTech, Inc. R.I. consults for Imagion Biosystems, a company developing imaging with magnetic iron oxide nanoparticles; and, for Magnetic Insight a company developing magnetic particle imaging technology. All other authors report no conflicts of interest.

\section{Ethics}

All animal studies were approved by the Institutional Animal Care and Use Committee at Johns Hopkins University.

\section{$\underline{\text { References }}$}

1. Hedayati, M., Abubaker-Sharif, B., Khattab, M., Razavi, A., Mohammed, I., Nejad, A., Wabler, M., Zhou, H., Mihalic, J., Gruettner, C., DeWeese, T. and Ivkov, R. (2018). An optimised spectrophotometric assay for convenient and accurate quantitation of intracellular iron from iron oxide nanoparticles. Int J Hyperthermia 34(4): 373-381.

2. Korangath, P., Barnett, J. D., Sharma, A., Henderson, E. T., Stewart, J., Yu, S. H., Kandala, S. K., Yang, C. T., Caserto, J. S., Hedayati, M., Armstrong, T. D., Jaffee, E., Gruettner, C., Zhou, X. C., Fu, W., Hu, C., Sukumar, S., Simons, B. W. and Ivkov, R. (2020). Nanoparticle interactions with immune cells dominate tumor retention and induce $\mathrm{T}$ cell-mediated tumor suppression in models of breast cancer. Sci Adv 6(13): eaay1601.

3. Marchal, S., El Hor, A., Millard, M., Gillon, V. and Bezdetnaya, L. (2015). Anticancer drug delivery: An update on clinically applied nanotherapeutics. Drugs 75(14): 1601-1611.

4. Sheen, M. R., Lizotte, P. H., Toraya-Brown, S. and Fiering, S. (2014). Stimulating antitumor immunity with nanoparticles. Wiley Interdiscip Rev Nanomed Nanobiotechnol 6(5): 496-505.

5. Staats, J., Divekar, A., McCoy, J. P. and Maecker, H. T. (2019). Guidelines for Gating Flow Cytometry Data for Immunological Assays. In: McCoy, Jr J. (Ed.). In: Immunophenotyping. Methods in Molecular Biology, vol 2032. Humana, New York, NY. https://doi.org/10.1007/978-14939-9650-6_5. 
6. Wu, W., Wu, Z., Yu, T., Jiang, C. and Kim, W. S. (2015). Recent progress on magnetic iron oxide nanoparticles: synthesis, surface functional strategies and biomedical applications. Sci Technol Adv Mater 16(2): 023501.

7. Zanganeh, S., Hutter, G., Spitler, R., Lenkov, O., Mahmoudi, M., Shaw, A., Pajarinen, J. S., Nejadnik, H., Goodman, S., Moseley, M., Coussens, L. M. and Daldrup-Link, H. E. (2016). Iron oxide nanoparticles inhibit tumour growth by inducing pro-inflammatory macrophage polarization in tumour tissues. Nat Nanotechnol 11(11): 986-994. 